LETTERS

If you have a burning desire to respond to a paper published in EM, why not make use of our "rapid response" option?

Log on to our web site (www.emjonline.com), find the paper that interests you, and send your response via email by clicking on the "eletters" option in the box at the top right hand corner.

Providing it isn't libellous or obscene, it will be posted within seven days. You can retrieve it by clicking on "read eletters" on our homepage.

The editors will decide as before whether to also publish it in a future paper issue.

\section{Intentional overdose of warfarin in an adolescent: need for follow up}

A 15 year old boy was seen in our emergency department with a history of ingesting 50, 5 $\mathrm{mg}$ tablets and 100, $1 \mathrm{mg}$ tablets of warfarin. He had also taken 14, $300 \mathrm{mg}$ tablets of allopurinol. This was confirmed to be fairly accurate by counting the number of tablets missing. The boy was well and had no specific symptoms. His vital signs were normal. He was admitted for close observation. His initial INR was 1.1; there were no signs of any superficial or internal bleeding.

As would be predicted with such a large ingestion his INR gradually rose to a peak of 5.0 after three days (see fig 1). He was given fresh frozen plasma $15 \mathrm{ml} / \mathrm{kg}$ on two occasions 24 hours apart. He also had $10 \mathrm{mg}$ of intravenous vitamin $\mathrm{K}$ injection twice on days 1 and 5. His INR came to normal values after five days and he was discharged home after several assessments and a major input from the psychiatric team. He did not show any signs of bleeding or any other adverse effects at any time. His vital signs remained stable throughout the hospital admission. His liver function, bone profile and full blood count remained normal and unchanged.

Oral anticoagulants inhibit the normal clotting process by interfering with the vitamin $\mathrm{K}$ dependent clotting factors (II, VII, IX, and X). On account of the differences in the degradation rates of these factors the therapeutic effects of warfarin are usually delayed by $8-16$ hours after oral or intravenous doses. ${ }^{1}$

Allopurinol interferes with the hepatic degradation of oral anticoagulants hence increasing the risk of bleeding.

There are several reports in the literature of accidental ingestion of rodenticides, which contain superwarfarin compounds. ${ }^{23}$ It has been shown in a recent review that these in general do not merit investigations or follow up. ${ }^{4}$ However, there are very few reports of

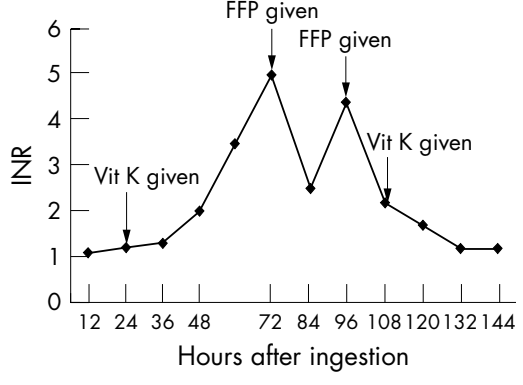

Figure 1 Graph showing INR over time (after warfarin ingestion).

intentional warfarin overdoses and none where allopurinol aggravated the damage. Our case report describes a boy with intentional overdose, which did cause serious alterations of his coagulation state. This report emphasises the need for serial prothrombin times in children who present with large intentional ingestion of warfarin even if they are completely asymptomatic.

A V Ramanan, P Gissen, B Bose-Haider Department of Paediatrics, Fairfield General Hospital, Bury BL9 7TD, UK

\section{References}

1 Goodman and Gilman's The pharmacological basis of therapeutics. 9th edn. New York: McGraw Hill, 1996:1347-50

2 Montanio CD, Wruk KM, Kulig KW, et a Acute pediatric warfarin (coumarin) ingestion: toxic effects despite early treatment. Am J Dis Child 1993:147:609-10.

3 Neguin C, Beuchee A, Pommereuil M, et al. Voluntary poisoning with a rodenticide in an adolescent. Arch Pediatr 1999;6:855-8.

4 Mullins ME, Brands CL, Daya MR.

Unintentional pediatric superwarfarin exposures: Do we really need a prothrombin time? Pediatrics 2000;105:402-4.

\section{Bilateral spontaneous \\ pneumothorax}

Wilkie et al describe a case of bilateral spontaneous pneumothorax in a 39 year old man. We are concerned that the conclusion drawn, namely the importance of urgent chest radiography for all patients in respiratory distress should not take precedence over the even more urgent need to ensure adequate oxygenation of the patient.

It appears clear that the patient was at significant risk of cardiorespiratory arrest despite stating that "cardiac output was not impaired."

We are told that despite only having an arterial oxygen saturation of $60 \%$ on 15 litres of oxygen and despite signs of significant hypoxaemia on blood gas analysis, the decision was made to proceed to radiography before intubation.

We would argue that prompt intubation should have preceded radiography, as delay in achieving oxygenation while awaiting the chest radiography could have had catastrophic consequences. 
We were also concerned at the one week follow up. The authors themselves state that grade 1 and 2 ankle sprains can take 10 days to recover to a level where the patient can return to work and yet the mean number of days off work was less than four in both groups. This would suggest that these were extremely mild ankle sprains and not reflective of the whole spectrum of this injury. Therefore the accuracy of grading the injuries acutely rather than at review several days later must also be questioned.

We feel that overall this study adds little to the literature on ankle sprain management, and certainly does not provide any evidence for a change of current accepted practice.

D Lewis

Department of Accident and Emergency Medicine Bedford NHS Trust, Kempston Road, Bedford MK42 9DJ, UK

P Atkinson

Department of Accident and Emergency Medicine, Luton and Dunstable NHS Trust, Lewsey Road, Luton

LU4 ODZ, UK

\section{References}

1 Watts BL and Armstrong B. Emerg Med J 2001;18:46-50.

2 Greenhalgh T. How to read a paper - The basis of evidence based medicine. London: BM Publishing Group, 1997.

\section{Authors' reply}

We welcome the opportunity to respond to the comments made by Drs Lewis and Atkinson on our recently published paper.'

Firstly, as our Consort diagram clearly shows, 85 patients declined randomisation, were not entered and therefore did not form part of the study population. We included this information to illustrate the clarity of ou study and because we felt it was interesting to note the number of patients who did not want to take part in a study aiming to recruit 400 patients. Our "drop out" rate was 203 of 400 patients-that is, $50.75 \%$. This weakness is covered fully in our discussion, where we pointed out that our study might be best considered as a pilot study. Even so our study, with 197 patients, is still the largest to tackle this research question. Previous studies have also suffered from disappointing follow up rates $^{2}$ and while using a funded research assistant may help, the fact remains that ankle sprains have a good prognosis ${ }^{34}$ with many patients not even seeking medical attention. ${ }^{5}$ Getting patients to consider their follow up telephone questionnaire or appoint ment as anything other than an inconvenience when they themselves feel significantly better is a difficult problem to solve.

We feel that Drs Lewis and Atkinson have missed the point when they criticise one of our chosen outcome measures. The fact that there was no difference between the groups is surely the point? We did find that significantly more analgesia was required in the double Tubigrip group and therefore obviously needed to discuss that finding. As regards the "crudeness" of that question, we return to the point made above on clarity. Our study was carried out without funding and with the invaluable help of non-medical A\&E receptionists. For the reasons above we chose not to bring patients back for follow up appoint ments but to telephone them with a standardised questionnaire instead. As anyone who has designed a questionnaire will know, the wording and reading age is vital and we had ours checked by an educational psychologist, hence the choice of such a basic question as "Did you take painkillers for your ankle injury?". Blister packs can get lost, patients may use them for other things or give them away and, of course, the patient needs to attend for follow up.

Do Drs Lewis and Atkinson routinely review all grade 1 and 2 ankle sprains for instability? In a study in which 150 patients with ankle sprains (not graded) were given no support but treated with elevation for 24 hours followed by motion exercise and weight bearing according to ability, $8 \%$ of patients had residual symptoms that they considered "inconvenient" at one year. ${ }^{6}$ The key to preventing chronic problems after ankle sprains is appropriate rehabilitation ${ }^{7}$ and the advice given to these patients is vital.

Drs Lewis and Atkinson also criticise our choice of follow up at one week and the fact that our patients seemed to return to work rather earlier than expected, suggesting that the accuracy of grading acute injuries rather than review several days later is questionable. However, the work used to quote anticipated recovery times for the different grades of injury was carried out almost a quarter of a century ago. ${ }^{8}$ As we said in our paper, the factors affecting patients' desire for an early return to work/ activities is complex but there have clearly been massive social changes over the past 25 years that are highly likely to influence these variables. We believe our patients were graded accurately and we continued reinforcing the grading system throughout the trial period. Also, deferring assessment and grading of sprains for several days is not an option as there is agreement that the excellent prognosis of these injuries means patients presenting more than 24 hours after injury should be excluded from trial entry.

We feel that Drs Lewis and Atkinson's final comments are unjustified. Research does not have to be (and probably never can be) perfect to be published, but what is vital is that the authors describe and explain their study's shortcomings. Our study was transparent and honest and remains the largest one in this area to date. We feel that by stimulating discussion we have certainly added something to this debate.

Finally, we find Drs Lewis and Atkinson's last line ironic. Where is the evidence to make double Tubigrip "accepted practice" in the first place?

Accident and Emergency Department, Queen Alexandra Hospital, Portsmouth, Hampshire PO6 3LY, UK B Armstrong

Emergency Department, Southampton General Hospital, Hampshire, UK Correspondence to: Dr Watts; Beverley.Watts@ukgateway.net

References

1 Watts BL, Armstrong B. A randomised controlled trial to determine the effectiveness of double Tubigrip in grade 1 and 2 (mild to moderate) ankle sprains. Emerg Med J 2001;18:46-50.

2 Brooks SC, Potter BT, Rainey JB. Treatment for partial tears of the lateral ligament of the ankle: a prospective trial. $B M$ 1981;282:606-7.

3 Ogilvie-Harris DJ, Gilbart M. Treatment modalities for soft tissue injuries of the ankle: a critical review. Clin J Sports Med 1995;5:175-86.

4 Karlsson J, Eriksson BI, Sward L. Early functional treatment for acute ligament injuries of the ankle joint. Scand J Med Sci Sports 1996;6:341-5.

5 Brostrom L. Sprained ankles: treatment and prognosis in recent ligament ruptures. Acta Chir Scand 1966;132:537-50.
6 Linde F, Hvass I, Jurgensen $U$, et al. Early mobilising treatment in lateral ankle sprains. Scand J Rehab Med 1986;18:17-21.

7 Renstrom PAFH, Konradsen L. Ankle ligament injuries. Br J Sports Med 1997; 3:11-20.

8 Brand RL, Black HM, Cox JS. The natura history of inadequately treated ankle sprain Am J Sports Med 1977;5:248-9.

\section{Effectiveness of double Tubigrip in grade 1 and 2 ankle sprains}

I was interested to read the article by Watts and Armstrong on a randomised controlled trial to determine the effectiveness of double Tubigrip in mild to moderate ankle sprains.

I agree completely and have shown in my previous research, which was quoted as a reference, that the mainstay of treatment in incomplete tears of the lateral ligament should be early mobilisation with pain relief as required. I am fully prepared to accept their findings that the use of double Tubigrip does not actually improve this healing process. However, to achieve the desired mobilisation I have foun in clinical experience that the application of a double Tubigrip support provides the patient with a feeling of comfort and confidence that allows the patient to begin this early mobilisation. I should be interested to know from the authors whether they experienced any difficulty in persuading patients to mobilise on a painful ankle without any apparent benefit of support or immediate pain relief being offered. Certainly there are many patients that I have seen who while totally "unable" to weightbear on initial presentation to the department after reassurance and the application of double Tubigrip were able to walk out immediately and thereafter made good progress. I think the move in all departments towards non-use of supportive bandaging will require a great dea of patient education to obtain their cooperation with the treatment or in their eyes lack of treatment.

S C Brooks

Accident and Emergency Department, Yeovil District Hospital, Yeovil, Somerset BA21 4AT, UK

\section{References}

1 Watts BL, Armstrong B. A randomised controlled trial to determine the effectiveness of double Tubigrip in grade 1 and 2 (mild to moderate) ankle sprains. Emerg Med J $2001 ; 18: 46-50$.

\section{Authors' reply}

We thank Dr Brooks for his comments on our paper. ${ }^{1}$ In answer to his question, we have consistently found that a full explanation of the nature of the injury, with advice on immediate and subsequent rehabilitation (how much to weight bear, elevation, ice treatment, and analgesia) and a statement as to why "those stretchy bandages" are not recommended, satisfies our patients with ankle sprains. This advice takes approximately one minute to give and is supplemented by a written advice card

We would like to point out that all the patients in our study were given analgesia on presentation and those who were genuinely unable to weight bear were given crutches to aid mobilisation. This is our standard practice and we would suggest that it is the reassurance and explanation that encourages those patients who are "unable" to weight bear initially to walk out of the department, rather than the "comfort" of a support bandage. Indeed we would like to draw attention to the finding in our study that the double Tubigrip group required significantly more analgesia than the 
control group. Although the factors responsible for this may be varied, we already know that the use of a cylindrical bandage where the ankle has a $90^{\circ}$ angle tends to produce a compressive band across the front of the joint, essentially causing a "tourniquet effect". Hence it is debatable how much "comfort" patients derive from this treatment and we feel that the potential for harm should not be dismissed.

Finally we would certainly agree with Dr Brooks that patient education is vital for any call for the cessation of routine double Tubigrip use to succeed. However, we suggest that this is easily and rapidly achievable at the initial presentation, by taking a little time to give a full explanation to the patient.

Accident and Emergency Department, Queen Alexandra Hospital, Portsmouth, Hampshire PO6 3LY, UK

B Armstrong

Emergency Department, Southampton General Hospital, Hampshire, UK

Correspondence to: Dr Watts

(Beverley.Watts@ukgateway.net)

\section{References}

1 Watts BL, Armstrong B. A randomised controlled trial to determine the effectiveness of double Tubigrip in grade 1 and 2 (mild to moderate) ankle sprains. Emerg Med J 2001:18;46-50.

\section{Volar metacarpophalangeal joint dislocation}

We read with interest the very rare case repor of Lam et al on volar metacarpophalangeal joint dislocation.

However, the subluxed and locked MCP joint is often confused with a dislocation of the MCP joint. We have seen three similar cases in our department over the past few years.

For example, a 78 year old former miner was referred to us by his general practitioner

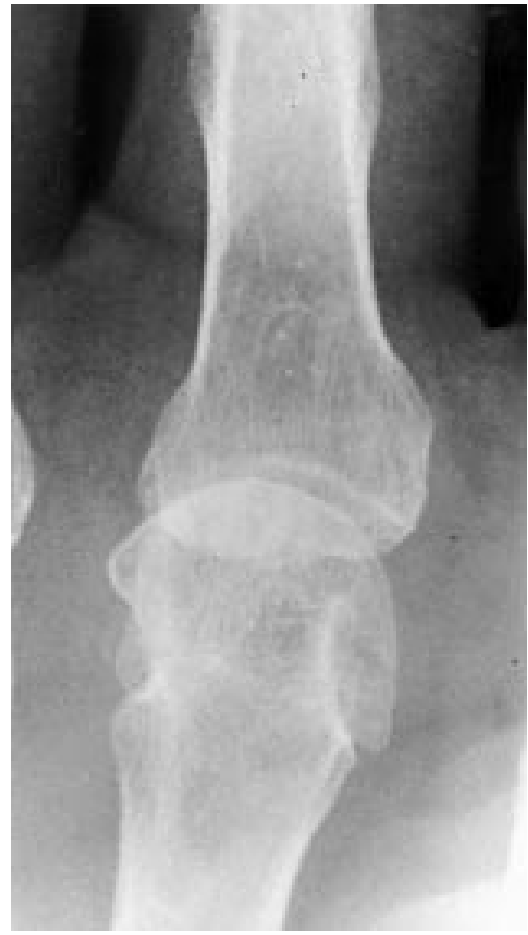

Figure 1 Radiograph of left middle finger showing subluxation of the MCP joint with marginal osteophytes.

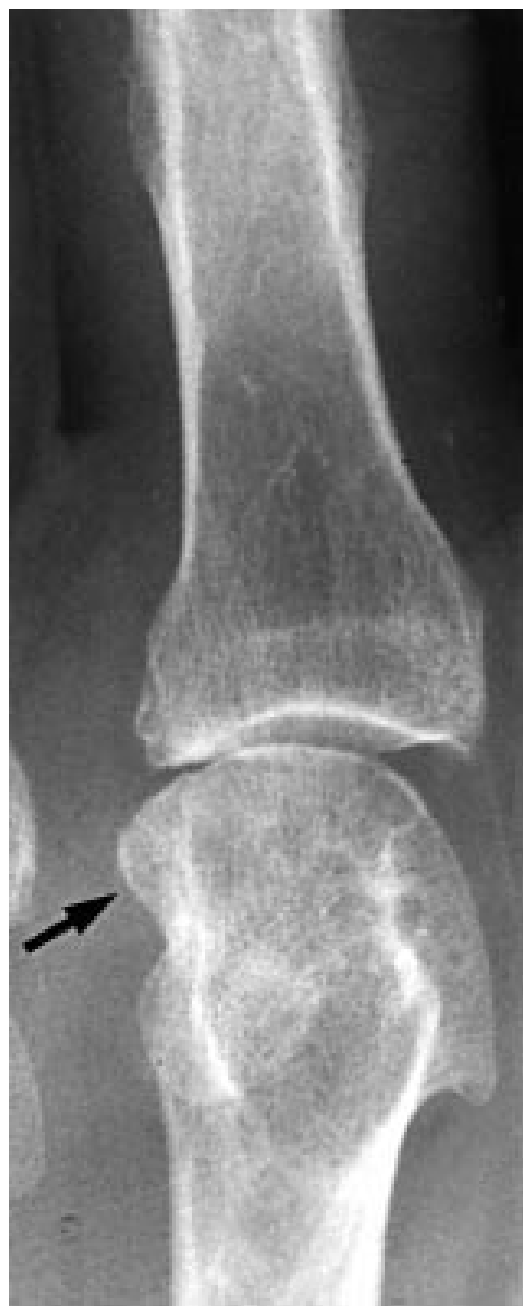

Figure 2 Post-manipulation check radiograph showing normal joint space with marginal osteophytes.

with a suspected dislocated MCP joint of the left middle finger. He had spontaneously developed pain in the finger two days previously. He was unable to move it. Past medical history included diabetic macular degeneration, myocardial infarction, ruptured aortic aneurysm, and hypertension. He was taking aspirin, nifedipine and glibenclamide.

On examination, the left middle finger was in flexed position at about $40^{\circ}$ and he was unable to extend it. There was no swelling. He was unable to flex the MCP joint any further. There was slight movement of the PIP and DIP joint, actively and passively. The PIP and DIP joints were in full extension. Radiographs of the left middle finger showed volar subluxation of the MCP joint, with margina osteophytes (fig 1). Under an intermetacarpal block with lignocaine (lidocaine) 1\%, $2 \mathrm{ml}$ on each side, the MCP joint was relocated with click. The patient was able to move the joint freely after the manipulation. A check radiograph revealed a normal joint space (fig 2) The patient was treated with neighbour strapping and advised to do active exercises after three days. He was reviewed four weeks later, there was a full range of movements of the finger. There was no tenderness and the patient had resumed his gardening.

A locked MCP joint is an unusual entity, characterised by a moderate flexion deformity. It must be differentiated from stenosing flexor tenosynovitis and volar dislocation of the MCP joint. Most commonly, the index and middle fingers are involved because of their prominent metacarpal heads. The condition is generally manifested in one of two ways, degenerative and spontaneous. ${ }^{2}$ In the former group the patients generally tend to be older and radiographs will, as in this case, usually reveal marginal osteophytes. ${ }^{3}$ Middle fingers are generally affected. In the spontaneous group, the patients are younger, and the condition tends to affect the index finger with the pathological structure being a very prominent radial condylar margin of the metacarpal head impaling the lateral collateral ligament ${ }^{2}$

It is difficult to recognise all these conditions in a busy emergency department, and when in doubt, a trial of gentle manipulation should be tried before referral to the hand surgeon for an operation.

P Muthu, A Fraser-Moodie Acciddent and Emergency Department, Derbyshire Royal Infirmary, London Road, Derby DE I 2QY, UK

\section{References}

1 Lam WL, Fitzgerald AM, Hooper G. Volar metacarpophalangeal joint dislocation. $J$ Accid Emerg Med 2000 17:226-8.

2 Glickel SZ, Barron AO, Eaton RG. Dislocations and ligament injuries in the digits. Green's operative hand surgery. 4th ed. London: Churchill Livingstone, 1999:786.

3 Stewart GJ, Williams EA. Locking of the metacarpophalangeal joints in degenerative disease. The Hand 1981:13:147-51.

4 Guly HR, Azam MA. Locked finger treated by manipulation. J Bone Joint Surg 1982:64B:73-5.

\section{Authors' reply}

We note with interest the comments made by Muthu and Fraser-Moodie. They discuss the occurrence of a locked metacarpophalangeal joint that is usually caused by snagging of the collateral ligaments over an adjacent osteophyte in older patients, which is obviously a condition that should be brought to the attention of accident and emergency staff. The main differential diagnosis being a trigger finger.

However, it is an entirely different condition to the one in our case report, ${ }^{1}$ which is a serious injury and one that is associated with serious and characteristic radiological changes requiring the early input of the hand surgery service.

A Fitzgerald, W L Lam, G Hooper Department of Hand Surgery, St John's Hospital, Livingston EH54 6PP, UK

Correspondence to: Mr Fitzgerald laidanfitzg@aol.com

\section{References}

1 Lam WL, Fitzgerald AM, Hooper G. Volar metacarpophalangeal joint dislocation. J Accid Emerg Med 2000;17:226-8.

\section{Calcium salts in management of hyperkalaemia}

In response to the letter by Dr McMorran ${ }^{1}$ we would like to comment on the use of calcium salts in the management of hyperkalaemia.

It is worth reiterating that the two formulations of intravenous calcium have distinct properties. The amount of elemental calcium $\left(\mathrm{Ca}^{2+}\right)$ is $8.9 \mathrm{mg} / \mathrm{ml}$ as $10 \%$ calcium gluconate and $27.2 \mathrm{mg} / \mathrm{ml}$ as $10 \%$ calcium chloride solution. The availability of $\mathrm{Ca}^{2+}$ may be limited in the calcium gluconate because of chelation, 
until hepatically metabolised. In haemodynamic instability with poor liver perfusion this might prove significant, hence the recommendation for use of the chloride salt in cardiac arrest. It should be noted, however, that several studies have failed to show differences in availability of ionised calcium even in the anhepatic stage of liver transplantation. ${ }^{2}$ Although the literature does not provide a definitive answer, we recommend that the chloride salt be used thereby eliminating concern over the possible impact of hepatic metabolism of the gluconate, and avoiding the confusion inherent in having two formulations with different doses on the resuscitation trolley.

M Davey, D Caldicott

Emergency Department, Royal Adelaide Hospital, Australia

Correspondence to: Dr M Davey, Emergency Department, Royal Adelaide Hospital, Adelaide, South Australia; mdavey@mail.rah.sa.gov.au

\section{References}

1 McMorran S. Treatment of hyperkalaemia in the emergency department. Emerg Med J $2001 ; 18: 233$

2 Martin T, Kang Y, Robertson K, et al. lonization and hemodynamic effects of calcium chloride and calcium gluconate in the absence of hepatic function. Anaesthesia 1990;73:62-5.

\section{BOOK REVIEWS}

\section{Accident and emergency: theory into practice}

Edited by B Dolan, L Holt. (Pp 564; £35.95) Baillère Tindall, 2000. ISBN 0-702-02239-X.

I was delighted to be asked to review this book as on the first superficial glance both the format and content looked very appealing. It is a wide ranging book covering both the clinical and professional needs of nurses in the accident and emergency department. It also seeks to uncover the theoretical and research basis for current day practice.

In the clinical sections, problems are approached from an anatomical and physiological view, giving a sound basis for the assessment and management of cases. I particularly liked the clear anatomical diagrams, with just enough detail. I would have found the addition of some radiograph useful as they are of particular relevance. The book covers the whole case mix of any department, approached from both a systems approach and from an age banded approach.

I did feel that certain sections were written from a theoretical viewpoint rather than from experience of current clinical practice. In particular, in the section preschool children on viral croup it was suggested that nebulised adrenaline (epinephrine) was the first line treatment. This is clearly not needed in the mild cases and should only be used in the severe cases when the child is to be admitted and will possibly need intensive care. It can cause dangerous rebound stridor and facilities for intubation must be ready.

The section on abdominal emergencies was good, but the assessment of abdominal trauma by primary and secondary survey was mixed up and very confusing not following the ATLS guidelines. The patient was log rolled in both the primary and secondary survey.

The section on minor head injuries was excellent. This is an area that often gets too little attention considering the number of patients presenting. The sections on pain management and violence and aggression were well written and useful, as was the whole section on psychological problems.

I found it odd for the section on nurse triage to be so far into the book, as it is the first contact most patients have with a nurse whatever their condition. In view of the importance of the nurses role and responsibility in this area, the section was a little lost.

The sections on Practice and Professional Issues overall, however, were excellent detailing clearly many conflicting issues in an A\&E nurses role. An example was the thoughtful discussion on the need for precise and accurate patient assessment, but in a limited time with limited resources.

Overall, the clinical areas of the book will be very useful as a bench reference book for nurses and the other areas are excellent to help nurses consider the breadth of professional issues they meet. I would recommend it to my nursing colleagues and also to my medical colleagues to give them some insight into the problems their nursing colleagues meet every working day.

\section{P O Brennan}

\section{Fibrinolytic and antithrombotic therapy: theory, practice and management}

R C Becker. (Pp 756; £39.95). Oxford University Press, 2000. ISBN 0-195-12331-X

Developments in fibrinolytic and antithrombotic therapy are among the most important recent advances in emergency medicine. This book provides a comprehensive guide from basic principles to clinical practice. Although it may fit into a (large) pocket, this is very much a reference book. The writing is precise and economical, largely unencumbered by conjecture or opinion, allowing vast amounts of factual information to be conveyed. While this creates impressive scope and depth it does little to engage the reader.

Part I covers the basic science, detailing concepts and diseases vaguely familiar from tortured nights of MRCP revision (and many others that weren't). If you agree that "The prevention and cost-effective management of thrombotic disorders requires a knowledge of fundamental pathobiologic principals..." you will find this section valuable. I must admit that I questioned the relevance of such knowledge the first time I learnt it. As it seems to have suffered disuse atrophy with clinical practice, I remain unconvinced.

The pharmacological agents are the focus of part II. Data from clinical trials are reproduced with merciless efficiency but little additional interpretation or explanation. Given that such data might rapidly be accessed electronically, I couldn't help feeling that an opportunity had been missed to provide either some guarantee of systematic secondary data collection, or some narrative to lighten the read. Part III (acute coronary syndromes: fundamental concepts) initially used a physiological principle, the open vessel hypothesis, to illustrate practical management issues with good effect. Unfortunately, chapter 18 plunged us back into a tabulated list of clinical trials.

The second half (parts IV to VI) examines practical management issues. Tables and algorithms are provided to assist the reader. The tables often seem to have been constructed from a pathological, rather than clinical, perspective. Lists are comprehensive but not ordered, and common items may even be neglected. For example, the top three differential diagnoses of chest pain are aortic dissection, expanding thoracic aneurysm and aortic intramural haematoma, while gastrooesophageal reflux and anxiety are not listed. The algorithms, particularly those involving risk stratification, present a practical and innovative approach to clinical management, but many require adaptation to the more conservative, and resource limited NHS.

In terms of factual data per square inch of paper, this book is truly awesome. However, A\&E clinicians facing the challenge of keeping up to date with a wide array of rapidly changing health technologies will probably prefer a more selective and more readable text.

S Goodacre

\section{Trauma: practical application of recent advances}

British Medical Bulletin vol 55, no 4. Edited by David W Yates. (Pp 947; £34.95). Royal Society of Medicine Press, 1999. ISBN 1-85315-440-7.

In his book the editor has given an overview of the varied nature of trauma and its effect on the individual and society. He has complemented this with individual chapters on specific injuries and their management where scientific advances have improved treatment and outcome as determined by evidence based practice.

Throughout the book, whether dealing with individual body system trauma, multiple casualties or the consequences of trauma, the need is highlighted for an organisational approach to trauma care with the development of strategies to deal with injury and its associated complications. In the relevant chapters there is discussion on the underlying biological mechanisms involved in injury and healing with reference to outcome. However, a comprehensive understanding is not possible within the scope of this book.

The chapters on epidemiology, which show its still depressing trends in association with resource allocation, injury prevention, comorbid factors and injury in the aging population complemented the book. Many of these subjects are often neglected or poorly written and it was refreshing to note that these were included.

The specialised chapters on trauma care were well written and interesting despite their specialist nature. Although of minimal interest to the generalist, they would be a valuable asset for those people who have to treat specific injuries.

In these times when the trauma epidemic shows no signs of cessation, and with an increase in the aging populations who are susceptible not only to injury but to the consequences of injury, there is a need for both accident prevention and development of rehabilitation. This has been covered comprehensively in this book.

Overall the editor has succeeded in covering trauma in a global fashion, taking the reader through the prehospital phase, through acute management and into the field of rehabilitation in an interesting way. He has combined the historical, developmental and factual components in a descriptive evidence based manner to make this book both interesting and informative reading. 
In this current atmosphere of resource allocation, prioritisation of care and risk management, those clinicians who practice evidence based medicine will find this book extremely useful.

This book would be useful for either trainees or consultants involved in the management of trauma care.

P A Evans

Leicester

\section{Self-assessment colour review of paediatric emergency medicine}

P O Brennan, J G Yassa, S Ludwig. (£15.95). Manson Publishing, 2001. ISBN 1-874-54546-4.

This is a fun book to have on your shelf! This book comprises 209 picture quizzes and answers, the pictures being well illustrated, full colour plates containing a mixture of common and not so common peadiatric conditions. The contributors are international from Australia, Uganda, Birmingham and Sheffield. The pictures relate to paediatric conditions including surgical, medical, metabolic, ophthalmological and dental conditions. Some of the cases are not commonly seen in UK practice, for example the 10 year old who sustained a penile tip injury due to a gunshot wound, or another example of a child sustaining an accident due to his arm becoming trapped in a wringer!

Overall this book is well written and contains few non-English phrases; however, when they do occur they are somewhat odd. An example might be "the outlook for the testis is superio if the hernia can be manually reduced!". However, this is to be somewhat pedantic abou what is a very handy and useful book certainly for study towards MRCPCH Part II but will be of interest to all clinicians who encounter children.
I must confess to having had a thoroughly enjoyable evening simply looking through at a wide variety of different conditions some common, some rare. I think the authors have succeeded in producing a well illustrated and informative addition to paediatric emergency medicine.

I Maconochie

St Mary's Hospital, London

\section{How to present at meetings}

Edited by G M Hall. (Pp 88; £12.95). BM Books, 2001. ISBN 0-727-991572-X

We have all attended poor presentations a meetings and most of us have gained our revenge by presenting poorly. In an effort to break this cycle of retribution Hall et al have produced their book How to present at meetings. This text is described as providing "a basic framework around which a proficient talk can be built" and there is no doubt that it succeeds in its aim. This short, clear and humorous book is written by a number of accomplished speakers, each of whom attempt to explain how to give a presentation that will be memorable for all the right reasons. The authors obviously write from experience and their explanations and advice are often illustrated through humorous examples of their own mistakes. This concept is taken to its logical conclusion with a whole chapter dedicated on how to get your "bad" presentation just right (or is that "just wrong?")

Fortunately the authors take their own advice and have produced a collection of brief, clear chapters, which make for a very readable book. This format means that it can be picked up in a spare five minutes or read completely within an hour depending on circumstances. The book is aimed at those speaking at formal meetings but the advice is as relevant to local teaching as it is to an international conference.

There is plenty of advice on preparation of your presentation and emphasis on its importance so if you are looking for short cuts you will not find them in this book. There are also chapters on the style of presentation and delivery as well as advice on stage presence from one of medicines many performers Alan Maryon Davis. The two chapters on visual aids are welcome and should be compulsory reading for anyone who ever steps to the front of a lecture theatre. One of these chapters is given over entirely to computer generated slides and will hopefully bring relief to those of us who develop a nervous twitch at the sight of spinning letters and the sound of a racing engine! Sandy Macara gives advice on dealing with questions from the audience but also on dealing with newspaper, radio and television interviews.

There is no doubt that a book like this should be required reading by anyone who makes a presentation and a departmenta copy for all staff to refer to would certainly be useful. My final recommendation, however should be that this book is posted to anyone presenting at a BAEM conference: it would improve the quality of presentation and guarantee we all get to coffee and biscuits on time

S McCormick

Northern General Hospital Sheffield

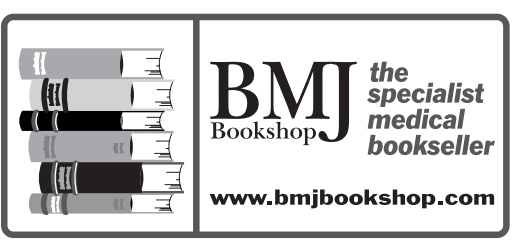

